\title{
Substance Use End Date Time
}

National Cancer Institute

\section{Source}

National Cancer Institute. Substance Use End Date Time. NCI Thesaurus. Code C83419.

The date and time substance usage has concluded. 